\title{
CD review
}

-

Special Care Dentistry: an interactive learning programme for the dental team Produced by J. Fiske MBE and R. Davies Smile-on Ltd

Copies of the CD can be obtained from Smile-on Ltd on 0207400 8989, or email info@smile-on.com. There is a small charge of $£ 25+$ VAT for further copies.

Launched by Dr Janice Fiske at the BDA 2006 conference, the rationale for the production of this Special Care Dentistry interactive learning programme is that the majority of people requiring special care dentistry can, and should, be treated in general dental practice. The aim of the e-learning programme is twofold. Firstly, to remove the stereotypes and myths that can surround people with disability; secondly, to encourage the dental team to provide care for people with disability by providing them with knowledge, information and practical tips. The programme encourages the user to question their values and attitudes, as well as their knowledge; it is underpinned by the team approach and the concept of 'shared care'.

The CD comes with an explanatory booklet, setting out the background, target audience (GDPs and their teams in the UK), aims and objectives, programme structure and system requirements. The programme starts with a clear menu of contents, comprising:

- Introduction

- Older people

- Medical conditions

- Physical disabilities

- Learning disabilities.

Navigation is easy, and the combination of audio, text and pictures is effective - there are no fussy graphics for the sake of it, or excessive blocks of text to read off-screen; vitally, the content is also clinically sound. For those users who want to research particular areas more deeply by surfing the net, there is a 'resources' button, which

\section{Competition}

Win a year's subscription to the Journal of Disability and Oral Health worth £120. Just answer the following question and return the answer form below to Competitions, Vital, BDJ Editorial, Nature Publishing Group, 4-6 Crinan Street, London, N1 9XW.

\section{Question}

According to the cover story in this issue of Vital (pages 39-41), how many people in the UK have a learning disability?
A. 1.2 million
B. 9.5 million

\section{JDOH ANSWER FORM}

Please PRINT your details below:

Title

First Name

\section{Last Name}

\section{Address}

\section{City}

Postal Code

\section{Email}

Job title

\section{Answer (please tick $\boldsymbol{V}$ ):}

\section{A $\square \quad B$}

If you prefer not to be cont
on to any third parties.

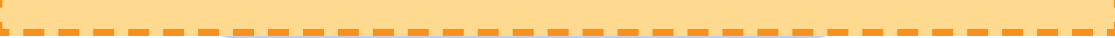

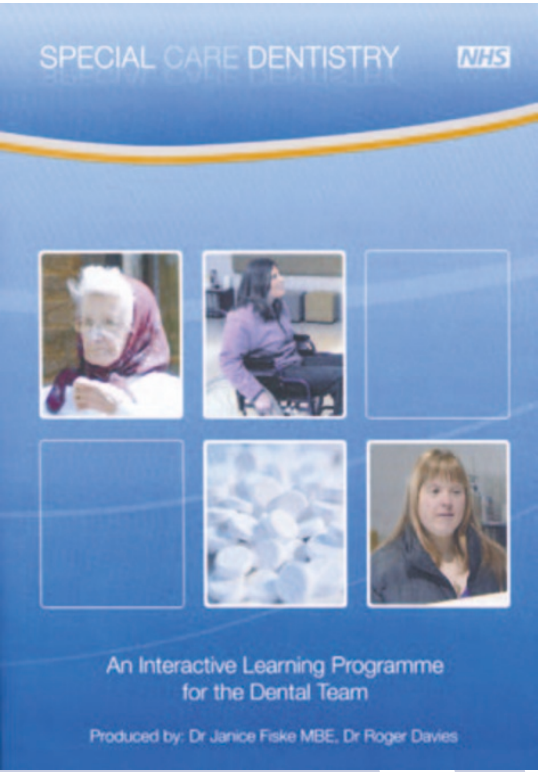

provides links to a number of websites, such as the British Society for Disability and Oral Health, and the British Society for Gerodontology. This option also gives access to some downloadable documents, including brief oral healthcare guidelines for older people, and a leaflet on dental care and autism.

Testing the programme, Dr Ronnie Levine (GDP) commented that he experienced technical problems with his iMAC - with his computer freezing repeatedly. He was also concerned about the appropriateness of some of the photographs; the denture in the older people section is vulcanite, which have not been made since the 1950s; the photo of an individual with type 2 diabetes, which the voice-over says affects middle-aged and elderly people, shows a young man.

These reservations apart, however, dental teams who have received one of the 16,000 free copies of this programme will find working through it rewarding, as it is a very useful and indeed essential guide to medically and physically compromised people. Completion of the programme results in participants being awarded CPD points.

With thanks to Dr Janice Fiske MBE and Dr Ronnie Levine OBE for their kind assistance.

Catherine Stillman-Lowe

Independent Oral Health Promotion Adviser 